\title{
Vasohibin-1 inhibits angiogenesis and suppresses tumor growth in renal cell carcinoma
}

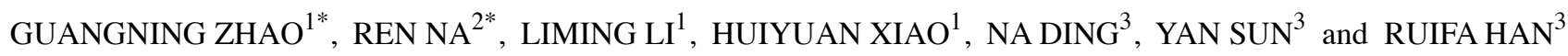 \\ ${ }^{1}$ Department of Urology, Tianjin Medical University General Hospital, Tianjin 300052; \\ ${ }^{2}$ Department of Clinical Laboratory, Tianjin Children's Hospital, Tianjin 300074; ${ }^{3}$ Department of Urology, \\ Second Hospital of Tianjin Medical University, Tianjin Institute of Urology, Tianjin 300211, P.R. China
}

Received November 7, 2016; Accepted May 30, 2017

DOI: $10.3892 /$ or.2017.5746

\begin{abstract}
Vasohibin-1 (VASH1) has recently been isolated as a novel inhibitor of angiogenesis. Several studies have demonstrated that VASH1 plays important roles in tumor angiogenesis but the role of this angiogenic inhibitor in renal cell carcinoma (RCC) has not been elucidated. We previously reported that VASH1 expression is reduced and is associated with clinicopathological features in RCC. In the present study, we investigated the biological effects of VASH1 in RCC by evaluating the effects of VASH1 on cell proliferation, cell cycle distribution, cell apoptosis and cell invasion in human umbilical vein endothelial cells (HUVECs) and 786-0 cells, and evaluating the effect of VASH1 on the growth of 786-0 cells in nude mice. A pReceiver-M61-VASH1 was transfected into HUVECs and 786-0 cells, and the expression level of VASH1 protein was examined by western blotting. Cell proliferation was detected by MTT assay, and cell cycle and apoptosis of HUVECs and 786-0 cells were analyzed by flow cytometry. The invasive ability of 786-0 cells was tested by Transwell assay. Finally, nude mouse models were established to evaluate the therapeutic effect of VASH1. The pReceiverM61-VASH1 effectively induced the expression of VASH1 in HUVECs and 786-0 cells. VASH1 overexpression effectively inhibited cell proliferation, arrested the cell cycle in the G0/ G1 phase and promoted cell apoptosis of HUVECs and 786-0 cells. VASH1 overexpression effectively inhibited the subcutaneous growth of 786-0 tumors in vivo. Therefore, VASH1 is a potential molecular-targeted therapy for patients with RCC.
\end{abstract}

Correspondence to: Dr Guangning Zhao, Department of Urology, Tianjin Medical University General Hospital, 154 Anshan Road, Heping, Tianjin 300052, P.R. China

E-mail: zgn123316979@126.com

*Contributed equally

Key words: renal cell carcinoma, vasohibin-1, angiogenesis, tumor inhibition, molecular-targeted therapy

\section{Introduction}

Renal cell carcinoma (RCC) is the most common neoplasm of the kidney in adults accounting for $2-3 \%$ of all adult malignancies and $80-90 \%$ of primary malignant renal tumors $(1,2)$. Currently, surgical resection is considered the first choice for treating RCC when possible and multiple treatments can be used together. However, relapse occurs in $20-40 \%$ of patients after curative nephrectomy, and most patients with RCC are relatively refractory to both systemic chemotherapy and radiotherapy (2). Moreover, the absence of biomarkers for the early detection and follow-up of the disease complicate the promptness and accuracy of diagnosis. Therefore, it is important to develop novel tumor markers that have higher sensitivity and reliability and effective therapeutic methods for treating RCC.

Tumor angiogenesis has been indicated to be a promising target for developing effective treatments for cancer patients. To date, many pro-angiogenic factors and angiogenic inhibitors have been identified, including growth factors, cytokines and proteases (3). Among the pro-angiogenic factors, vascular endothelial growth factor (VEGF), platelet-derived growth factor (PDGF) and hypoxia-inducible factor (HIF) families have been demonstrated to play important roles in mediating tumor angiogenesis, and are associated with tumor progression, invasion, metastasis and poor survival of patients with RCC (4-7). However, the role of angiogenic inhibitors in RCC development is poorly understood.

Vasohibin-1 (VASH1), as a novel endothelium-derived inhibitor of angiogenesis, has been recently identified (8). VASH1 expression is induced in response to angiogenic stimuli such as VEGF-A and fibroblast growth factor (FGF)-2 and can inhibit angiogenesis in an autocrine manner $(8,9)$. Studies have demonstrated that VASH1 plays important roles in disease-induced angiogenesis $(10,11)$, and malignancies including breast cancer, gynecological and hepatocellular carcinoma (HCC), gastric, lung and colon cancer (12-18). In addition, various preliminary studies have demonstrated that VASH1 exerts an antitumor effect by inhibiting angiogenesis in the tumor environment (19-22). However, the expression pattern of VASH1 and its potential clinical and biological roles in RCC have not been elucidated.

In our previous study, we firstly investigated the expression pattern of VASH1 in RCC samples by immunohistochemistry. 
We found that VASH1 was expressed in both RCC tissues and adjacent non-tumorous renal tissues (ANRT). VASH1 expression was reduced in the RCC tissues compared to that in ANRT, and its expression showed a correlation with clinicopathological features of RCC (23). Based on the expression pattern of VASH1 in RCC, we postulated that VASH1 may not only act as an intrinsic angiogenesis inhibitor produced by $\mathrm{ECs}$, but also plays a critical role in regulating angiogenesis as an extrinsic factor secreted by other cells in RCC.

In the present study, we sought to determine whether VASH1 decreased angiogenesis and suppressed tumor growth in RCC. We demonstrated that overexpression of VASH1 effectively inhibited cell proliferation, arrested the cell cycle in the G0/G1 phase and promoted cell apoptosis in HUVECs and 786-0 cells in vitro and inhibited the subcutaneous growth of 786-0 tumors in vivo. Therefore, according to knowledge based on other angiogenesis inhibitors, our findings have implications for the potential use of VASH1 as a candidate molecular-targeted therapy for patients with RCC.

\section{Materials and methods}

Cell culture and cell transfection. Human umbilical vein endothelial cells (HUVECs) were obtained from the Tianjin Institute of Urology. HUVECs were maintained at $37^{\circ} \mathrm{C}$ in a humidified atmosphere of $5 \% \mathrm{CO}_{2}$ in RPMI-1640 medium (Gibco, Grand Island, NY, USA), supplemented with $10 \%$ fetal bovine serum (FBS) (Gibco, Montevideo, Uruguay), penicillin $(100 \mathrm{U} / \mathrm{ml})$ and streptomycin $(100 \mu \mathrm{g} / \mathrm{ml})$. The human renal carcinoma cell line 786-0 was obtained from Tianjin Institute of Urology. 786-0 cells were maintained at $37^{\circ} \mathrm{C}$ in a humidified atmosphere of 5\% $\mathrm{CO}_{2}$ in RPMI-1640 medium, supplemented with 10\% FBS (both from Gibco), penicillin (100 U/ml) and streptomycin $(100 \mu \mathrm{g} / \mathrm{ml})$. Plasmid pReceiverM61-VASH1 was successfully constructed in our laboratory at the Tianjin Institute of Urology. Each cell line was transiently transfected using Lipofectamine 2000 (Invitrogen, Carlsbad, CA, USA), and an empty vector was used to ensure equal content in transfections.

Western blot analysis. Forty-eight hours after transfection, the VASH1 protein expression in HUVECs and 786-0 cells was determined using western blot analysis. Cells were rinsed twice with cold phosphate-buffered saline (PBS), then, homogenized in RIPA cell lysate and centrifuged. The protein concentration of each sample was quantified by the Bradford assay. Total cell extract protein $(50 \mathrm{mg})$ was separated by $10 \%$ SDS-PAGE and transferred onto polyvinylidene fluoride (PVDF) membrane. After being blocked with 5\% skimmed milk for $1 \mathrm{~h}$ at room temperature, the membrane was incubated with goat anti-vasohibin-1 (Santa Cruz Biotechnology, Santa Cruz, CA, USA) antibody overnight at $4^{\circ} \mathrm{C}$. After extensive washes, blots were incubated with a dilution $(1: 2,000)$ of horseradish peroxidase-conjugated anti-goat or anti-goat IgG (Santa Cruz Biotechnology) for $90 \mathrm{~min}$ at $37^{\circ} \mathrm{C}$. The bands were developed with 3,3'-diaminobenzidine (DAB). GAPDH (Santa Cruz Biotechnology) was used as an internal control. We used the ratio of the grey value of VASH1 and GAPDH as a variable for statistical analysis.
Cell proliferation assay. The effect of VASH1 on the proliferation of HUVECs and 786-0 cells was measured using MTT assay. Briefly, the cells were seeded in 96-well plates at a density of $1 \times 10^{5} /$ well and cultured for $24 \mathrm{~h}$. Then, the cells were divided into pReceiver-M61-VASH1, pReceiver-M61 or blank control group. After transfection at different time points, $20 \mu \mathrm{l}$ of $5 \mathrm{mg} / \mathrm{ml}$ MTT solution was added to each well. After $4 \mathrm{~h}$ of incubation at $37^{\circ} \mathrm{C}$, the supernatant was removed and $150 \mu \mathrm{l}$ of dimethyl sulfoxide (DMSO) was added to each well, and shaken for $10 \mathrm{~min}$. Absorbance of each well was measured on a microplate reader at a wavelength of $490 \mathrm{~nm}$. All experiments were carried out in triplicate.

Cell cycle assay. The effect of VASH1 on the cell cycle distribution of HUVECs and 786-0 cells was identified using flow cytometric analysis. The cells were plated in 6-well plates at a density of $1 \times 10^{5} /$ well and cultured at $37^{\circ} \mathrm{C}$ in a $5 \%$ $\mathrm{CO}_{2}$ incubator for $24 \mathrm{~h}$. Then, the cells were divided into a pReceiver-M61-VASH1, pReceiver-M61 or blank control group. Forty-eight hours after transfection, the adherent cells were collected by $0.25 \%$ trypsinization, washed in PBS and centrifugated. Cells were resuspended at $1 \times 10^{6}$ cells $/ \mathrm{ml}$ in $\mathrm{PBS}$ and fixed in ice-cold ethanol overnight at $4^{\circ} \mathrm{C}$. Fixed cells were centrifuged and washed once with PBS. Each sample was resuspended in propidium iodide (PI) solution $(33 \mu \mathrm{g} / \mathrm{ml} \mathrm{PI}$, $0.13 \mathrm{mg} / \mathrm{ml}$ RNase A, $10 \mathrm{mmol} / 1$ EDTA, 0.5\% Triton X-100). Samples were analyzed using a fluorescence-activated cell sorting (FACS) flow cytometer (BD Biosciences, San Jose, CA, USA), and DNA histograms were analyzed with modified software. Each test was repeated in triplicate.

Cell apoptosis assay. The effect of VASH1 on the apoptosis of HUVECs and 786-0 cells was identified using flow cytometric analysis. Cells were plated in 6-well plates at a density of $1 \times 10^{5} /$ well and cultured at $37^{\circ} \mathrm{C}$ in a $5 \% \mathrm{CO}_{2}$ incubator for $24 \mathrm{~h}$. Then, they were divided into a pReceiver-M61VASH1, pReceiver-M61 or blank control group. Forty-eight hours after transfection, the adherent cells were collected by $0.25 \%$ trypsinization, washed in PBS and centrifuged and resuspended at $1 \times 10^{6}$ cells $/ \mathrm{ml}$ in PBS. Then, the cells were stained with FITC-labeled Annexin V (10 $\mu \mathrm{l})(20 \mu \mathrm{g} / \mathrm{ml})$ and PI $(5 \mu \mathrm{l})(50 \mu \mathrm{g} / \mathrm{ml})$ and immediately analyzed using a fluorescence-activated cell sorting (FACS) flow cytometer (BD Biosciences). The results were analyzed with modified software. Each test was repeated in triplicate.

Cell invasion assay. The effect of VASH1 on 786-0 cell invasion was assessed using a 24-well Transwell insert (pore size, $8 \mu \mathrm{m}$; Corning, Corning, NY, USA). The cells were divided into pReceiver-M61-VASH1, pReceiver-M61 or blank control group. Forty-eight hours after transfection, the cells were collected by $0.25 \%$ trypsinization, washed in PBS and centrifuged and resuspended at $1 \times 10^{6}$ cells $/ \mathrm{ml}$. Cells were starved in serum-free medium overnight, and $2 \times 10^{4}$ cells/well were resuspended in $200 \mu \mathrm{l}$ serum-free medium and placed in the upper chamber with $8-\mu \mathrm{m}$ filter pores. The membrane undersurface was coated with Matrigel (BD Biosciences) mixed with RPMI-1640 serum-free medium in a 1:5 dilution for $30 \mathrm{~min}$ at $37^{\circ} \mathrm{C}$. The lower chamber was filled with $600 \mu \mathrm{l}$ $10 \% \mathrm{FBS}$ as the chemoattractant. After incubation for $48 \mathrm{~h}$, 
A

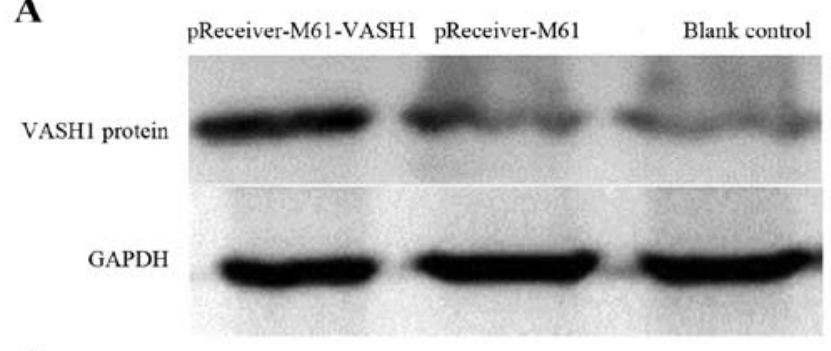

C

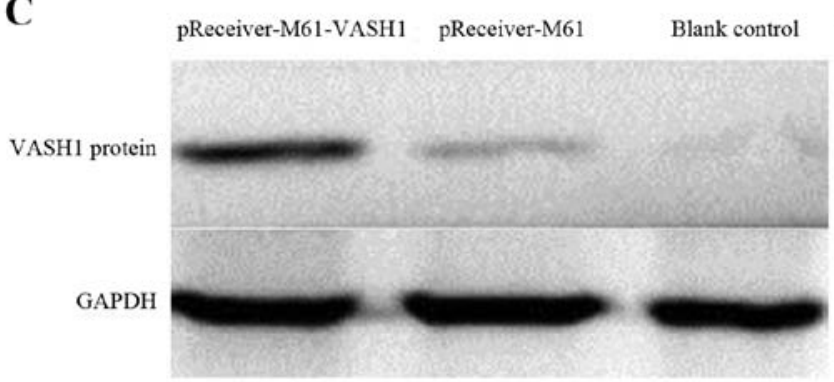

B

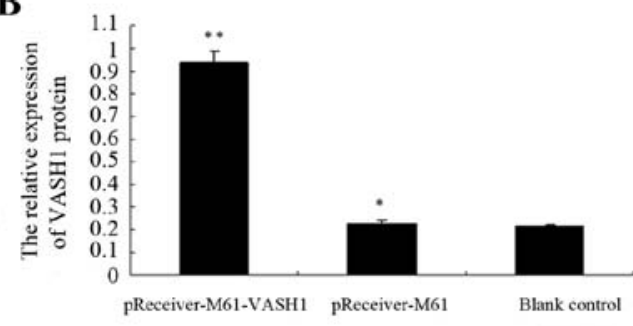

D

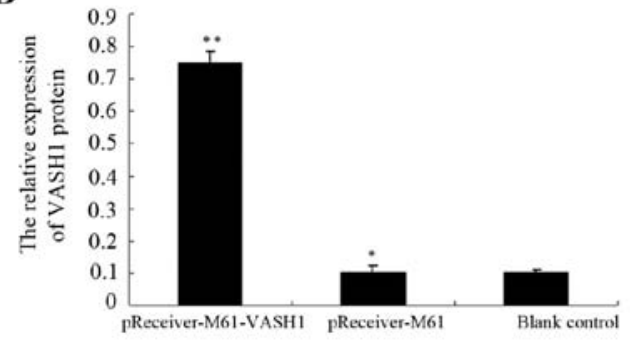

Figure 1. Western blot analysis of VASH1 protein expression after cell transfection. (A) Western blot analysis of VASH1 protein expression in the different HUVEC groups. (B) The increased protein level of VASH1 in the pReceiver-M61-VASH1-transfected cells compared to the pReceiver-M61-transfected cells and blank control cells in the HUVEC group. (C) Western blot analysis of VASH1 protein expression in the different 786-0 cell groups. (D) The increased protein level of VASH1 in the pReceiver-M61-VASH1-transfected cells compared to the pReceiver-M61-transfected cells and blank control cells in the 786-0 cell group. GAPDH is shown as an internal control; ${ }^{* *} \mathrm{P}<0.05,{ }^{*} \mathrm{P}>0.05$ as compared with the blank control.

non-migrated/non-invaded cells were removed from the upper well with cotton swabs while the migrated/invaded cells were then fixed with methanol, stained with $0.1 \%$ crystal violet, and photographed (magnification, $\mathrm{x} 200$ ) in five independent fields for each well. Each test was repeated in triplicate.

In vivo antitumoral activity. Six-week-old male BALB/c nude mice were purchased from the Animal Resources Centre, Military Medical Sciences (Beijing, China). All animal experiments were approved by the Tianjin Medical University (Tianjin, China) Ethics Committee, and carried out in accordance with the NIH Guide for the Care and Use of Laboratory Animals. All animals were kept under specific pathogen-free, temperature-controlled conditions and handled in accordance with the Institutional Animal Welfare Guidelines. Mice were randomly divided into three groups $(n=6)$ : one group received an injection of 786-0 cells transfected with pReceiver-M61-VASH1; one group received an injection of 786-0 cells transfected with pReceiver-M61 as the negative control; another group received an injection of $786-0$ cells as a non-treated control group. Cells $\left(5 \times 10^{6}\right)$ in a volume of $200 \mu 1$ were injected into the left flank area of the nude mice. The tumor volume was measured with a caliper at day $7,14,21,28$ and 35 , respectively. The tumor volume (V) was calculated at regular intervals according to the formula: $\mathrm{V}=\pi / 6 \mathrm{x}$ length $\mathrm{x}$ width $^{2}$. These three groups were treated for 35 days. Afterward, the mice were sacrificed, and tumors were extracted.

Statistical analysis. Statistical analysis was performed using SPSS software (version 16.0). The significance of differences between multiple groups was determined by one-way analysis of variance. Data are expressed as means \pm standard deviation. Statistical significance was set at $\mathrm{P}<0.05$.

\section{Results}

Expression of VASHI after transfection of the HUVECs and 786-0 cells. To evaluate whether the recombinant plasmids were successfully transfected into the HUVECs and 786-0 cells and whether VASH1 protein was expressed, we investigated the protein expression of VASH1 in HUVECs and 786-0 cells after transfection with pReceiver-M61-VASH1. In the HUVECs, the relative protein expression of VASH1 $(0.936 \pm 0.053)$ was significantly higher in the pReceiver-M61-VASH1 group, when compared with the level in the blank control $(0.214 \pm 0.008)$ and pReceiver-M61 group $(0.227 \pm 0.013)(\mathrm{P}<0.05)$ (Fig. 1A and B). In the 786-0 cells, the relative protein expression of VASH1 $(0.751 \pm 0.035)$ was significantly higher in the pReceiver-M61VASH1 group, when compared with the level in the blank control $(0.104 \pm 0.007)$ and pReceiver-M61 group $(0.102 \pm 0.024)$ $(\mathrm{P}<0.05)$ (Fig. 1C and D). These results showed that transfection with pReceiver-M61-VASH1 increased the VASH1 protein level in the HUVECs and 786-0 cells.

Effect of VASH1 on the cell growth of HUVECs and 786-O cells. To investigate the effect of VASH1 overexpression on the cell growth of HUVECs and 786-0 cells, the level of cell proliferation was assessed by MTT assay after transfection. In HUVECs, the optical density (OD) of the pReceiver-M61VASH1, pReceiver-M61 and blank control group at $24 \mathrm{~h}$ after transfection was $0.239 \pm 0.026,0.269 \pm 0.021$ and $0.265 \pm 0.028$, respectively. There was no significant difference among the three groups $(\mathrm{P}>0.05)$. The OD of the pReceiver-M61-VASH1, pReceiver-M61 and blank control group $48 \mathrm{~h}$ after transfection was $0.315 \pm 0.012,0.403 \pm 0.026$ and $0.423 \pm 0.010$, respectively. The OD of the pReceiver-M61-VASH1, pReceiver-M61 and blank control group at $72 \mathrm{~h}$ after transfection was $0.431 \pm 0.017,0.632 \pm 0.025,0.661 \pm 0.039$, respectively. The OD 
A

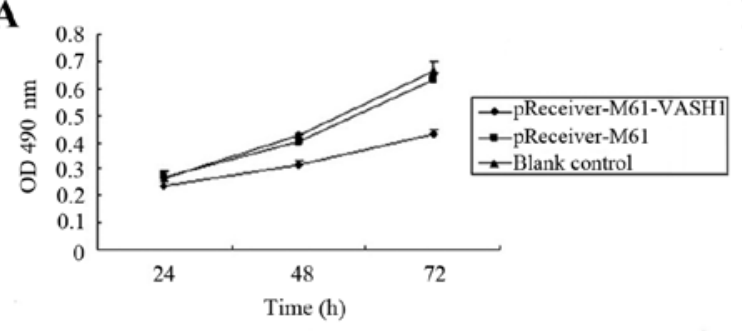

C

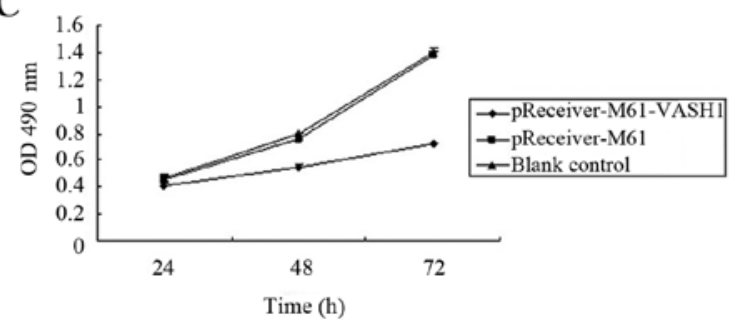

B

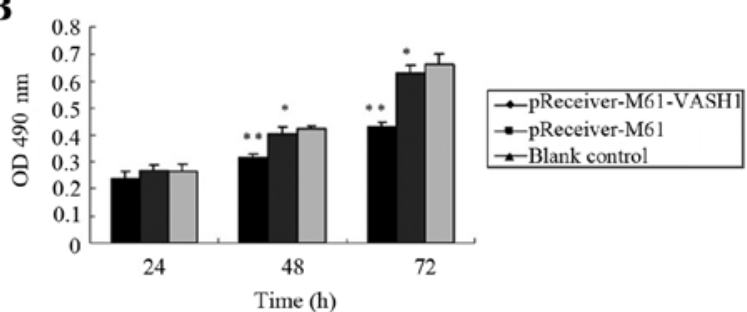

D

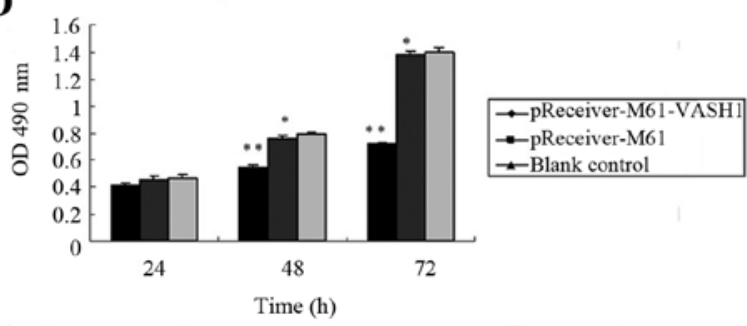

Figure 2. VASH1 overexpression decreases the proliferation of HUVECs and 786-0 cells. (A and B) MTT assay showing that VASH1 overexpression significantly reduced the proliferation of HUVECs over a 48-h period in vitro. (C and D) MTT assay showing that VASH1 overexpression significantly reduced the proliferation of $786-0$ cells over a 48 -h period in vitro; ${ }^{* *} \mathrm{P}<0.05,{ }^{*} \mathrm{P}>0.05$ as compared with the blank control.

of the pReceiver-M61-VASH1 group was significantly lower compared to that of the pReceiver-M61 or blank control group at 48 and $72 \mathrm{~h}$ after transfection $(\mathrm{P}<0.05)$. There was no significant difference between the pReceiver-M61 and blank control group ( $\mathrm{P}>0.05)$ (Fig. 2A and B). In the 786-0 cells, the OD of the pReceiver-M61-VASH1, pReceiver-M61 and blank control group at $24 \mathrm{~h}$ after transfection was $0.411 \pm 0.016$, $0.453 \pm 0.029$ and $0.469 \pm 0.028$, respectively. There was no significant difference among the three groups $(\mathrm{P}>0.05)$. The OD of the pReceiver-M61-VASH1, pReceiver-M61 and blank control group at $48 \mathrm{~h}$ after transfection was $0.547 \pm 0.022$, $0.756 \pm 0.026$ and $0.792 \pm 0.010$, respectively. The OD of the pReceiver-M61-VASH1, pReceiver-M61 and blank control group at $72 \mathrm{~h}$ after transfection was $0.718 \pm 0.017,1.384 \pm 0.025$ and $1.396 \pm 0.039$. The OD of the pReceiver-M61-VASH1 group was significantly lower compared to that of the pReceiver-M61 or blank control group at 48 and $72 \mathrm{~h}$ after transfection $(\mathrm{P}<0.05)$. There was no significant difference between the pReceiver-M61 and blank control group ( $\mathrm{P}>0.05$ ) (Fig. 2C and D). These results showed that overexpression of VASH1 significantly reduced the growth of HUVECs and 786-0 cells in vitro.

Effect of VASHI on the cell cycle distribution of HUVECs and 786-O cells. The effect of VASH1 on the cell cycle distribution of HUVECs and 786-0 cells after transfection was analyzed by flow cytometric analysis. At $48 \mathrm{~h}$ after transfection of HUVECs, the pReceiver-M61-VASH1 transfection group had an increase in the G0/G1 phase cells $(84.90 \pm 5.42 \%)(\mathrm{P}<0.05)$ as compared with the pReceiver-M61 $(63.68 \pm 8.62 \%)$ and blank control group $(56.89 \pm 2.35 \%)$, and a decrease in $\mathrm{S}$ phase cells $(13.99 \pm 5.39 \%)(\mathrm{P}<0.05)$ as compared with the pReceiver-M61 $(33.90 \pm 9.34 \%)$ and blank control group (42.52 $\pm 2.45 \%)$, respectively (Fig. 3A and B). At $48 \mathrm{~h}$ after transfection in the 786-0 cells, the pReceiver-M61-VASH1 group exhibited an increase in $\mathrm{G} 0 / \mathrm{G} 1$ phase cells $(77.91 \pm 4.89 \%)(\mathrm{P}<0.05)$ as compared with the pReceiver-M61 (59.55 $\pm 2.00 \%)$ and blank control group $(57.05 \pm 1.33) \%$, and a decrease in $\mathrm{S}$ phase cells
$(21.27 \pm 5.67 \%)(\mathrm{P}<0.05)$ as compared with the pReceiver-M61 $(40.13 \pm 1.73 \%)$ and blank control group $(42.60 \pm 1.28 \%)$, respectively (Fig. 3C and D). These results showed that overexpression of VASH1 had an effect on the cell cycle distribution of HUVECs and 786-0 cells. VASH1 arrested the cell cycle of the HUVECs and 786-0 cells in the G0/G1 phase in vitro.

Effect of VASH1 on the apoptosis of HUVECs and 786-O cells. The effect of VASH1 on cell apoptosis in the HUVECs and 786-0 cells after transfection was examined by FCM. At $48 \mathrm{~h}$ after transfection of the HUVECs, the percentage of apoptotic cells in the pReceiver-M61-VASH1 group was significantly higher $(5.06 \pm 0.39 \%)(\mathrm{P}<0.05)$ than that in the pReceiver-M61 $(1.41 \pm 0.26 \%)$ and blank control group $(0.83 \pm 0.31 \%)$, while there was no statistically significant difference between that in the pReceiver-M61 and the blank control group ( $\mathrm{P}>0.05)$ (Fig. $4 \mathrm{~A}$ and B). At $48 \mathrm{~h}$ after transfection in the 786-0 cells, the percentage of apoptotic cells in the pReceiver-M61-VASH1 group was significant higher $(13.09 \pm 1.39 \%)(\mathrm{P}<0.05)$ than that in the pReceiver-M61 $(4.47 \pm 0.48 \%)$ and blank control group $(3.24 \pm 0.39 \%)$, while there was no statistically significant difference between that in the pReceiver-M61 and blank control group ( $\mathrm{P}>0.05$ ) (Fig. 4C and D). These results showed that overexpression of VASH1 significantly promoted cell apoptosis in the HUVECs and 786-0 cells in vitro.

Effect of VASH1 on 786-O cell invasion. The effect of VASH1 on the invasive ability of the 786-0 cells after transfection was assessed by Transwell assay. At $48 \mathrm{~h}$ after transfection, the number of 786-0 cells passing through the EC Matrix gel in the pReceiver-M61-VASH1 group did not show significant differences $(65.33 \pm 8.16)$ compared with that in the pReceiver-M61 (68.87 \pm 7.44$)$ and blank control group $(69.53 \pm 7.59)(\mathrm{P}>0.05)$ (Fig. 5A and B). These results showed that overexpression of VASH1 did not reduce the invasiveness of the 786-0 cells in vitro. 
Aa

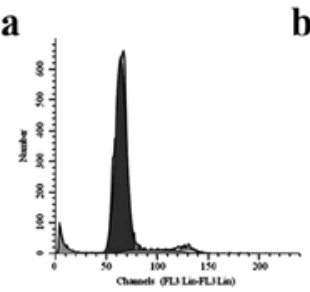

$\mathrm{Ca}$

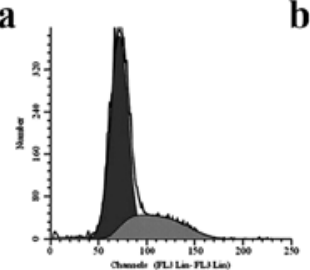

b

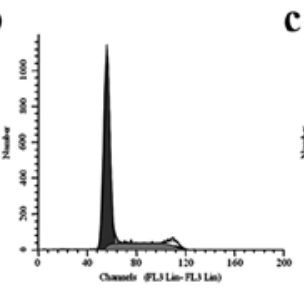

b

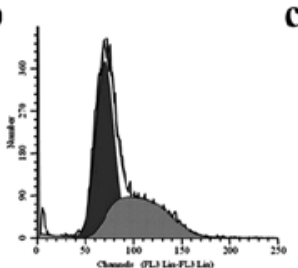

c

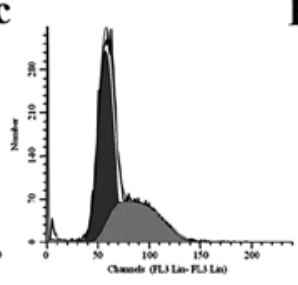

B

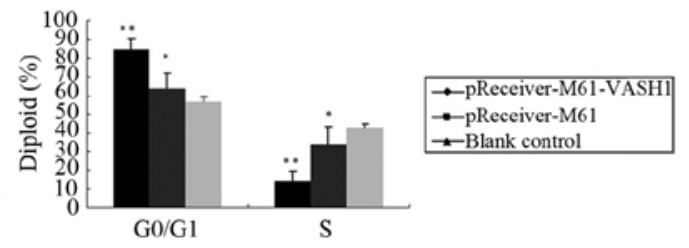

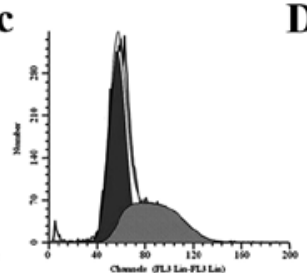

D

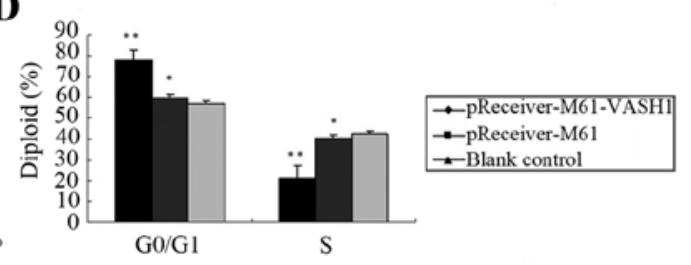

Figure 3. VASH1 overexpression induces G0/G1 phase cell cycle arrest of HUVECs and 786-0 cells. (A and B) Cell cycle analysis of the transfected HUVECs by FACS. Cells which were transfected with pReceiver-M61-VASH1 showed an increased G0/G1 phase population and a decreased S phase population compared with the pReceiver-M61-transfected and blank control cells (a, pReceiver-M61-VASH1-transfected group; b, pReceiver-M61-transfected group; c, blank control group). (C and D) Cell cycle analysis of the transfected 786-0 cells by FACS. Cells which were transfected with pReceiver-M61-VASH1 showed an increased G0/G1 phase population and a decreased S phase population compared with the pReceiver-M61-transfected and blank control cells (a, pReceiverM61-VASH1-transfected group; b, pReceiver-M61-transfected group; c, blank control group); ${ }^{* *} \mathrm{P}<0.05,{ }^{*} \mathrm{P}>0.05$ as compared with the blank control.

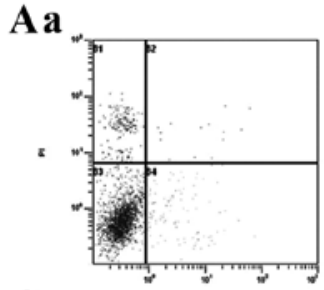

Ca

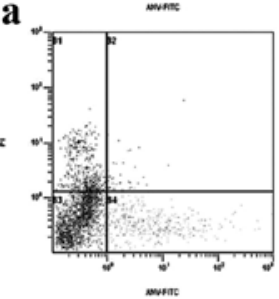

b

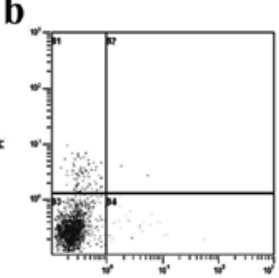

b

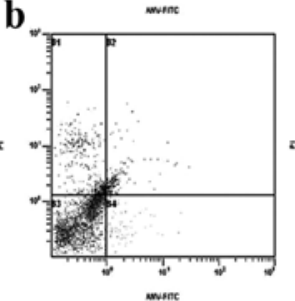

c

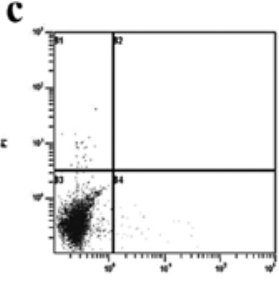

c

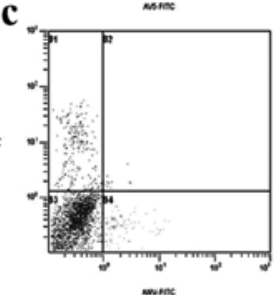

B

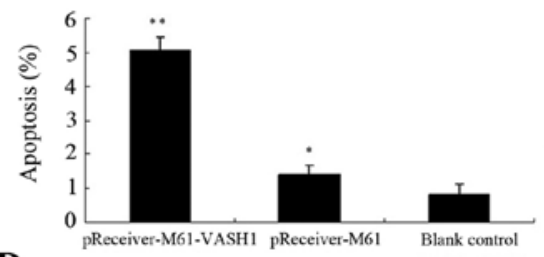

D

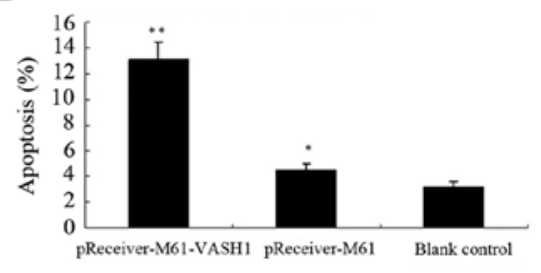

Figure 4. VASH1 overexpression induces HUVEC and 786-0 cell apoptosis. (A and B) Flow cytometric analysis showed that overexpression of VASH1 expression increased HUVEC apoptosis (a, pReceiver-M61-VASH1-transfected group; b, pReceiver-M61-transfected group; c, blank control group). (C and D) Flow cytometric analysis showed that overexpression of VASH1 increased 786-0 cell apoptosis (a, pReceiver-M61-VASH1-transfected group; b, pReceiverM61-transfected group; c, blank control group); ${ }^{* *} \mathrm{P}<0.05,{ }^{*} \mathrm{P}>0.05$ as compared with the blank control.
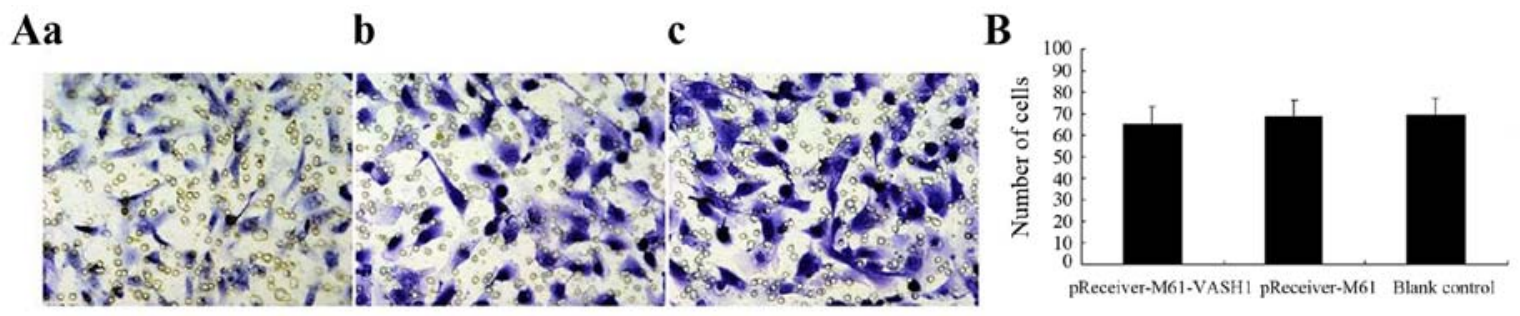

Figure 5. VASH1 overexpression does not reduce 786-0 cell invasion. (A and B) Invasion analysis of transfected 786-0 cells by Transwell assay at $48 \mathrm{~h}$ after transfection. Overexpression of VASH1 did not reduce 786-0 cell invasion (a, pReceiver-M61-VASH1-transfected group; b, pReceiver-M61-transfected group; c, blank control group).

Overexpression of VASH1 inhibits 786-0 tumor growth in vivo. To evaluate the efficacy of VASH1 overexpression in suppressing 786-0 tumor growth in an animal model, the tumor volume was measured. All mice survived during the period of treatment. After 35 days, the tumor volume of the pReceiver-M61-VASH1 group $\left(168.23 \pm 25.33 \mathrm{~mm}^{3}\right)$ 

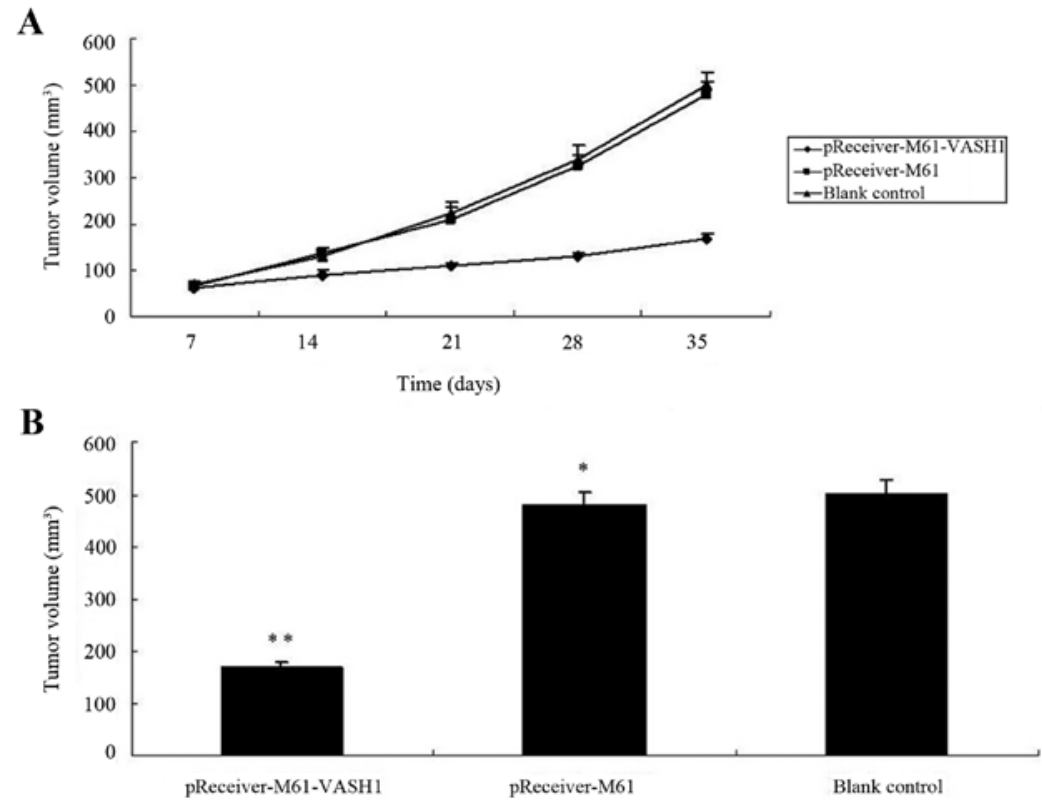

Figure 6. VASH1 overexpression inhibits 786-0 tumor growth in vivo. (A) The tumor growth curves indicated significant growth inhibition in the pReceiverM61-VASH1 group after 21 days, but no differences between the pReceiver-M61 and blank control group was noted. (B) After 35 days, the tumor volume from the pReceiver-M61-VASH1 group was significantly smaller than that from the pReceiver-M61 and blank control group; ${ }^{* *} \mathrm{P}<0.05$, ${ }^{*} \mathrm{P}>0.05$ as compared with the blank control.

was significantly smaller than that for the pReceiver-M61 and blank control group $(478.83 \pm 27.32$ and $500.67 \pm 28.21 \mathrm{~m}$ $\mathrm{m}^{3}$, respectively) $(\mathrm{P}<0.05)$, while there was no statistically significant difference between the pReceiver-M61 and blank control group $(\mathrm{P}>0.05)$. The tumor growth curves indicated significant growth inhibition in the pReceiver-M61-VASH1 group after 21 days, but no difference between the pReceiverM61 and blank control group was noted (Fig. 6A and B). These results showed that overexpression of VASH1 inhibited the subcutaneous growth of 786-0 tumors in vivo.

\section{Discussion}

Recently, angiogenesis has been indicated to be a pivotal event in various biological processes, and it plays a critical role in physiological and pathological processes particularly in multiple types of cancers $(24,25)$. Renal cell carcinoma (RCC) is a solid tumor that arises from the proximal convoluted tubules of the kidney and is characterized by abundant angiogenesis (26). In view of the resistance of RCC to most existing cytotoxic drugs or radiotherapy, therapies targeting the various molecules involving angiogenesis may be highly desirable. Tumor angiogenesis is induced when 'angiogenic homeostasis' is disrupted in tumor tissues (24). Therefore, it is reasonable to hypothesize that when we reconstruct the balance by applying agents to block stimulators or by applying exogenous angiogenesis inhibitors directly, the process of angiogenesis may be inhibited.

Vasohibin-1 (VASH1), as a novel endothelium-derived inhibitor of angiogenesis, has been recently identified (8). Its expression is induced in response to angiogenic stimuli such as VEGF-A, fibroblast growth factor (FGF)-2 and can inhibit angiogenesis in an autocrine manner $(8,9)$. Several previous studies have demonstrated that VASH1 as an angiogenesis inhibitor plays important roles in disease-induced angiogenesis such as nephropathy (10), retinal disease (11) and malignancies including breast cancer, gynecological and hepatocellular carcinoma (HCC), gastric, colon and lung cancer (12-18). In addition, various preliminary studies have demonstrated that VASH1 exerts an antitumor effect by inhibiting angiogenesis in the tumor environment (19-22).

Previous studies have shown that VASH1 is selectively expressed in the ECs of tumor tissue, and the expression of VASH1 in tumor tissue was found to be significantly increased when compared to that noted in normal tissue. Furthermore, the increase in expression of VASH1 strongly correlates with the advancement of the degree of malignancy $(12-14,16,17)$. However, we investigated the expression pattern of VASH1 and the association with clinicopathological features in RCC in our previous study (23). We found that VASH1 was expressed mainly in the cytoplasm and membrane of tumor cells and partly in vascular endothelial cells in RCC. In ANRT, it was mainly expressed in the cytoplasm and membrane of renal tubular epithelial cells and partly in vascular endothelial cells and glomerular mesangial cells. Based on the above findings and the literature which indicated that VASH1 mRNA is also expressed in a wide range of tissues and organs $(27,28)$, we hypothesized that VASH1 may not only act as an intrinsic angiogenesis inhibitor produced by ECs, but also may function as an extrinsic factor secreted by other cells to regulate the process of angiogenesis in RCC. In addition, we found that the expression level of VASH1 in RCC tissue was significant lower than that in ANRT and was significantly reduced with the increased degree of malignancy in RCC tissues. In addition, a significantly negative correlation was noted between VASH1 expression and HIF-1 $\alpha$ expression and a significantly negative correlation was noted between VASH1 expression and MVD in RCC (23). Therefore, VASH1 expression is reduced 
and is associated with clinicopathological features in RCC. Considering the seemingly paradoxical observations compared to that in other types of tumors, we presume that it may result from the difference in histological origin and the cancer type. It may also be attributed to the complexity and distinctiveness of the secretory pathway of VASH1 in different cancers.

In the present study, we investigated the biological effects of VASH1 by evaluating the effects of VASH1 on cell proliferation, cell cycle, cell apoptosis and cell invasion in HUVECs and 786-0 cells and evaluating the effect of VASH1 on the growth of 786-0 tumors in nude mice. MTT assay demonstrated that overexpression of VASH1 significantly reduced the growth of HUVECs and 786-0 cells in vitro. Flow cytometric analysis revealed that overexpression of VASH1 had an effect on the cell cycle distribution of HUVECs and 786-0 cells; VASH1 overexpression was able to arrest HUVECs and 786-0 cells in the G0/G1 phase in vitro. We also found that VASH1 overexpression significantly promoted HUVEC and 786-0 cell apoptosis in vitro. Nevertheless, overexpression of VASH1 did not reduce 786-0 cell invasion in vitro. Furthermore, a strong antitumor effect of VASH1 in vivo was observed, as tumor growth in nude mice with xenografts was significantly suppressed. Therefore, we hypothesized that disruption of 'angiogenic homeostasis' results in abundant angiogenesis, and the reduction in VASH1 expression in RCC tissues may partially explain the reason why there is abundant angiogenesis in RCC. In the present study by applying exogenous VASH1 directly, the process of angiogenesis in RCC was inhibited.

In recent years, molecular-targeted therapy has been clinically applied. Tyrosine kinase inhibitors (TKIs) including bevacizumab, sunitinib, sorafenib, pazopanib, axitinib and mTOR inhibitors such as temsirolimus and everolimus have achieved favorable clinical responses and have been administered as first-line or second-line therapy for patients with RCC (29). Nevertheless, since various angiogenic factors are involved in tumor angiogenesis, only targeting a single angiogenic factor may likely be ineffective. Therefore, to achieve sufficient therapeutic benefit, it may be necessary to simultaneously target multiple angiogenic factors. It was recently reported that VASH1 inhibits angiogenesis mediated by various angiogenic factors other than VEGF suggesting that VASH1, which acts alone to inhibit multiple angiogenic factors, is a more effective therapeutic agent compared with VEGF inhibitors in terms of improving patient survival (20).

Anti-angiogenic therapy has been approved for several types of cancers and several drugs are in clinical use. However, this type of drugs may have side-effects including hypertension or proteinuria due to the impairment of normal quiescent vessels. It was recently reported that VASH1 did not increase mean blood pressure and urinary albumin excretion in animal experiments (30). It has also been reported that VASH1 did not affect any morphological changes in normal blood vessels (31), wound healing, body weight and peripheral blood flow (32) in adenoviral VASH1 gene-treated mice. These findings all suggest that VASH1 could be a potential candidate anti-angiogenic therapy with fewer or less side-effects.

In summary, here we present both in vitro and in vivo evidence that VASH1 effectively inhibits the cell proliferation, arrests the cell cycle in the G0/G1 phase and promotes cell apoptosis in HUVECs and 786-0 cells and could suppress the subcutaneous growth of 786-0 tumors. Therefore, according to our knowledge of angiogenesis inhibitors, VASH1 could potentially be utilized as a candidate for molecular-targeted therapy for patients with RCC. Nonetheless, the effect of VASH1 on RCC needs to be verified in other RCC cell lines and investigation of the inhibitory effects of VASH1 on tumor models in situ is warranted. In addition, further studies are required to clarify the underlying inhibitory mechanisms and the targets of signal transduction pathways of anti-angiogenesis in RCC.

\section{Acknowledgements}

The present study was supported by grants from the Tianjin Medical University General Hospital Youth Foundation (award no. ZYYFY2014034; grant recipient, G.Z.).

\section{References}

1. Rini BI, Campbell SC and Escudier B: Renal cell carcinoma. Lancet 73: 1119-1132, 2009

2. Motzer RJ, Bander NH and Nanus DM: Renal-cell carcinoma. N Engl J Med 335: 865-875, 1996.

3. Carmeliet P and Jain RK: Angiogenesis in cancer and other diseases. Nature 407: 249-257, 2000

4. Qian CN, Huang D, Wondergem B and Teh BT: Complexity of tumor vasculature in clear cell renal cell carcinoma. Cancer 115 (Suppl 10): S2282-S2289, 2009.

5. Xu L, Tong R, Cochran DM and Jain RK: Blocking plateletderived growth factor-D/platelet-derived growth factor receptor beta signaling inhibits human renal cell carcinoma progression in an orthotopic mouse model. Cancer Res 65: 5711-5719, 2005

6. Sulzbacher I, Birner P, Träxler M, Marberger M and Haitel A: Expression of platelet-derived growth factor-alpha alpha receptor is associated with tumor progression in clear cell renal cell carcinoma. Am J Clin Pathol 120: 107-112, 2003.

7. Dorević G, Matusan-Ilijas K, Babarović E, Hadzisejdić I, Grahovac M, Grahovac B and Jonjić N: Hypoxia inducible factor-1 $\alpha$ correlates with vascular endothelial growth factor $\mathrm{A}$ and $\mathrm{C}$ indicating worse prognosis in clear cell renal cell carcinoma. J Exp Clin Cancer Res 28: 40, 2009.

8. Watanabe K, Hasegawa Y, Yamashita H, Shimizu K, Ding Y, Abe M, Ohta H, Imagawa K, Hojo K, Maki H, et al: Vasohibin as an endothelium-derived negative feedback regulator of angiogenesis. J Clin Invest 114: 898-907, 2004.

9. Sonoda H, Ohta H, Watanabe K, Yamashita H, Kimura H and Sato Y: Multiple processing forms and their biological activities of a novel angiogenesis inhibitor vasohibin. Biochem Biophys Res Commun 342: 640-646, 2006.

10. Nasu T, Maeshima Y, Kinomura M, Hirokoshi-Kawahara K, Tanabe K, Sugiyama H, Sonoda H, Sato Y and Makino H: Vasohibin-1, a negative feedback regulator of angiogenesis, ameliorates renal alterations in a mouse model of diabetic nephropathy. Diabetes 58: 2365-2375, 2009.

11. Shen J, Yang X, Xiao WH, Hackett SF, Sato Y and Campochiaro PA: Vasohibin is up-regulated by VEGF in the retina and suppresses VEGF receptor 2 and retinal neovascularization. FASEB J 20: 723-725, 2006.

12. Tamaki K, Moriya T, Sato Y, Ishida T, Maruo Y, Yoshinaga K, Ohuchi N and Sasano H: Vasohibin-1 in human breast carcinoma: A potential negative feedback regulator of angiogenesis. Cancer Sci 100: 88-94, 2009.

13. Yoshinaga K, Ito K, Moriya T, Nagase S, Takano T, Niikura H, Yaegashi $\mathrm{N}$ and Sato Y: Expression of vasohibin as a novel endothelium-derived angiogenesis inhibitor in endometrial cancer. Cancer Sci 99: 914-919, 2008.

14. Yoshinaga K, Ito K, Moriya T, Nagase S, Takano T, Niikura H, Sasano H, Yaegashi N and Sato Y: Roles of intrinsic angiogenesis inhibitor, vasohibin, in cervical carcinomas. Cancer Sci 102: 446-451, 2011.

15. Hosaka T, Kimura H, Heishi T, Suzuki Y, Miyashita H, Ohta H, Sonoda H, Moriya T, Suzuki S, Kondo T, et al: Vasohibin-1 expression in endothelium of tumor blood vessels regulates angiogenesis. Am J Pathol 175: 430-439, 2009. 
16. Wang Q, Tian X, Zhang C and Wang Q: Upregulation of vasohibin-1 expression with angiogenesis and poor prognosis of hepatocellular carcinoma after curative surgery. Med Oncol 29: 2727-2736, 2012.

17. Shen Z, Kauttu T, Seppänen H, Vainionpää S, Ye Y, Wang S, Mustonen $\mathrm{H}$ and Puolakkainen P: Vasohibin-1 and vasohibin-2 expression in gastric cancer cells and TAMs. Med Oncol 29: 2718-2726, 2012

18. Zhang T, Yu TT, Zhang DM, Hou XM, Liu XJ, Zhao D and Shan L: Vasohibin-1 expression detected by immunohistochemistry correlates with prognosis in non-small cell lung cancer. Med Oncol 31: 963, 2014.

19. Liu S, Han B, Zhang Q, Dou J, Wang F, Lin W, Sun Y and Peng G: Vasohibin-1 suppresses colon cancer. Oncotarget 6: 7880-7898, 2015.

20. Takahashi Y, Saga Y, Koyanagi T, Takei Y, Machida S, Taneichi A, Mizukami H, Sato Y, Matsubara S and Fujiwara H: The angiogenesis regulator vasohibin-1 inhibits ovarian cancer growth and peritoneal dissemination and prolongs host survival. Int J Oncol 47: 2057-2063, 2015.

21. Takahashi Y, Saga Y, Koyanagi T, Takei Y, Machida S, Taneichi A Mizukami H, Sato Y, Matsubara S and Fujiwara H: Vasohibin-1 expression inhibits advancement of ovarian cancer producing various angiogenic factors. Cancer Sci 107: 629-637, 2016.

22. Shen Z, Yan Y, Ye C, Wang B, Jiang K, Ye Y, Mustonen H, Puolakkainen $\mathrm{P}$ and Wang S: The effect of Vasohibin-1 expression and tumor-associated macrophages on the angiogenesis in vitro and in vivo. Tumour Biol 37: 7267-7276, 2016.

23. Zhao G, Yang Y, Tang Y, Han R and Sun Y: Reduced expression of vasohibin-1 is associated with clinicopathological features in renal cell carcinoma. Med Oncol 29: 3325-3334, 2012.
24. Folkman J: Tumor angiogenesis: Therapeutic implications. N Engl J Med 285: 1182-1186, 1971.

25. Folkman J: Role of angiogenesis in tumor growth and metastasis. Semin Oncol 29 (Suppl 16): S15-S18, 2002.

26. Mancilla-Jimenez R, Stanley RJ and Blath RA: Papillary renal cell carcinoma: A clinical, radiologic, and pathologic study of 34 cases. Cancer 38: 2469-2480, 1976.

27. Nimmagadda S, Geetha-Loganathan P, Pröls F, Scaal M, Christ B and Huang R: Expression pattern of Vasohibin during chick development. Dev Dyn 236: 1358-1362, 2007.

28. Naito H, Kidoya H, Sato Y and Takakura N: Induction and expression of anti-angiogenic vasohibins in the hematopoietic stem/progenitor cell population. J Biochem 145: 653-659, 2009.

29. Vitale MG and Cartenì G: Clinical management of metastatic kidney cancer: The role of new molecular drugs. Future Oncol 12: 83-93, 2016.

30. Miyashita H, Suzuki H, Ohkuchi A and Sato Y: Mutual balance between vasohibin-1 and soluble VEGFR-1 in endothelial cells. Pharmaceuticals 4: 782-793, 2011.

31. Heishi T, Hosaka T, Suzuki Y, Miyashita H, Oike Y, Takahashi T, Nakamura T, Arioka S, Mitsuda Y, Takakura T, et al: Endogenous angiogenesis inhibitor vasohibin1 exhibits broad-spectrum antilymphangiogenic activity and suppresses lymph node metastasis. Am J Pathol 176: 1950-1958, 2010.

32. Li D, Zhou K, Wang S, Shi Z and Yang Z: Recombinant adenovirus encoding vasohibin prevents tumor angiogenesis and inhibits tumor growth. Cancer Sci 101: 448-452, 2010. 